\title{
Characterization of Cider Apples on the Basis of Their Fatty Acid Profiles
}

\author{
Domingo Blanco-Gomis, ${ }^{*}, \dagger$ Juan J. Mangas Alonso, ${ }^{\ddagger}$ \\ Inmaculada Margolles Cabrales,$^{\dagger}$ AND Pilar Arias Abrodo ${ }^{\dagger}$ \\ Departamento de Química Física y Analítica, Universidad de Oviedo, E-33006 Oviedo, Spain, and \\ Servicio Regional de Investigación y Desarrollo Agroalimentario (S.E.R.I.D.A.) \\ Principado de Asturias, E-33300 Villaviciosa, Spain
}

\begin{abstract}
In the current study, the fatty acids composition of 30 monovarietal apple juices from six cider apple varieties belonging to two categories was analyzed. The different apple juices were obtained from three consecutive harvests $(1997,1998$, and 1999). The fatty acids concentration in apple juice together with chemometric techniques such as principal components analysis (PCA), soft independent modeling of class analogy (SIMCA), and linear discriminant analysis (LDA), allowed us to differentiate apple juices on the basis of the sweet or sharp category to which the cider apple variety belongs. Fatty acids such as the unsaturated oleic and linoleic acids, and saturated caprylic, capric, stearic, and palmitic acids were related to the sweet cider apple category, while pentadecanoic acid is related to the sharp class.
\end{abstract}

KEYWORDS: Fatty acids; cider apple; chemometrics

\section{INTRODUCTION}

The apple crop and the making of processed apple products (apple juice, cider, brandy, etc.) represents the third source of income of Asturias (northern Spain) after animal productions such as milk and meat. Therefore, knowledge of the chemical composition of each apple variety and the effect of blending them is of interest, so as to obtain higher quality processed apple products.

It is well-known that fatty acids play an important role on the sensory quality of foods. They contribute to flavor as precursors of volatile compounds such as C6-aldehydes and C6alcohols (1) by means of the action of oxygen and a multienzymatic system made up of lipoxygenase, hydroperoxide lyase, and alcohol dehydrogenase. Fatty acids can also be converted into flavor and fragrance products by means of microbial systems through several routes of oxidation, such as $\alpha$-oxidation, $\beta$-oxidation, production of methyl ketones, $\varpi$-oxidation with formation of macrocyclic lactones, sub-terminal oxidation, and midchain oxidation (1). At the same time, caprylic and capric acid and their ethyl esters play an important role in the spontaneous stoppage of alcoholic fermentation before the complete consumption of fermentable sugars, since they are yeast inhibitors $(2,3)$.

The higher fatty acids, such as linoleic and oleic acid, may be involved in the foam inhibition phenomenon (4). Consequently, the control of fatty acids might be of interest because

* To whom correspondence should be addressed.

† Universidad de Oviedo.

$\doteqdot$ Servicio Regional de Investigación y Desarrollo Agroalimentario (S.E.R.I.D.A.). of their effect on foam formation and stability, as the ability of cider to form foam is an important feature in terms of product attractiveness to the consumer. In the case of cider, excessive and nonconsistent lacing and/or high foam stability cause a decrease in sensory assessment. It is therefore important for the cider producer to control the level of fatty acids in apple juice, as they might affect the foam characteristics of the cider produced.

Fatty acids have also been used to characterize food. The analysis of minor and major fatty acids in almonds allowed Spanish almond varieties to be characterized $(5,6)$ and Italian blood orange juices were differentiated by means of their fatty acids composition (7). Likewise, the use of a fatty acid profile allowed ciders made from apples harvested in the region of Asturias to be differentiated from those made from apples harvested outside of this region (8).

The aim of this study was therefore to determine the fatty acid composition of 30 apple juices made from cider apples of different varieties in order to ascertain whether these analytes allowed sweet and sharp varieties to be differentiated. This could enable the cider producer to better control the raw material, thus establishing an adequate apple mixture so as to control the development of the fermentative process and to obtain products with a high sensory quality.

\section{MATERIALS AND METHODS}

Chemicals. Individual fatty acids of a purity of over $98 \%$ were purchased from Sigma (St. Louis, MO). Solvents, hexane for organic trace analysis, and chloroform were obtained from Merck (Darmstadt, Germany) and methanol from Romil (Cam- 
Table 1. Mean Values $(\mathrm{mg} / \mathrm{L})$ and Standard Deviations of Sharp and Sweet Apple Varieties

\begin{tabular}{|c|c|c|c|c|c|c|}
\hline \multirow[b]{2}{*}{ fatty Acid } & \multicolumn{2}{|c|}{ sweet } & \multicolumn{4}{|c|}{ sharp } \\
\hline & Coloradona & Verdialona & Durona de Tresali & Raxao & Solarina & Xuanina \\
\hline caprylic (C8) & $0.60 \pm 0.03$ & $0.45 \pm 0.04$ & $0.24 \pm 0.03$ & $0.56 \pm 0.04$ & $0.49 \pm 0.05$ & $0.34 \pm 0.06$ \\
\hline capric (C10) & $0.27 \pm 0.01$ & $0.20 \pm 0.04$ & $0.24 \pm 0.04$ & $0.16 \pm 0.02$ & $0.21 \pm 0.01$ & $0.11 \pm 0.03$ \\
\hline lauric (C12) & $0.23 \pm 0.05$ & $0.23 \pm 0.03$ & $0.22 \pm 0.02$ & $0.31 \pm 0.04$ & $0.17 \pm 0.02$ & $0.14 \pm 0.03$ \\
\hline myristic (C14) & $0.43 \pm 0.07$ & $0.46 \pm 0.02$ & $0.53 \pm 0.03$ & $0.62 \pm 0.02$ & $0.31 \pm 0.02$ & $0.19 \pm 0.06$ \\
\hline pentadecanoic (C15) & $0.15 \pm 0.06$ & $0.23 \pm 0.04$ & $0.23 \pm 0.03$ & $0.24 \pm 0.03$ & $0.20 \pm 0.03$ & $0.30 \pm 0.02$ \\
\hline palmitic (C16) & $4.90 \pm 0.26$ & $4.59 \pm 0.15$ & $4.03 \pm 0.38$ & $2.46 \pm 0.84$ & $5.69 \pm 0.27$ & $4.18 \pm 0.22$ \\
\hline palmitoleic $\left(\mathrm{C} 16: 1^{\Delta 9}\right)$ & $0.09 \pm 0.01$ & $0.13 \pm 0.02$ & $0.06 \pm 0.03$ & $0.12 \pm 0.05$ & $0.11 \pm 0.01$ & $0.06 \pm 0.04$ \\
\hline stearic (C18) & $4.60 \pm 0.06$ & $5.18 \pm 0.43$ & $4.66 \pm 0.35$ & $4.29 \pm 0.09$ & $4.93 \pm 0.31$ & $3.12 \pm 0.26$ \\
\hline oleic $\left(C 18: 1^{\Delta 9}\right)$ & $0.38 \pm 0.09$ & $0.29 \pm 0.03$ & $0.44 \pm 0.03$ & $0.33 \pm 0.09$ & $0.29 \pm 0.03$ & $0.28 \pm 0.05$ \\
\hline linoleic $\left(C 18: 2^{\Delta 9,12}\right)$ & $0.22 \pm 0.09$ & $0.10 \pm 0.04$ & $0.09 \pm 0.01$ & $0.07 \pm 0.03$ & $0.06 \pm 0.03$ & $0.07 \pm 0.01$ \\
\hline
\end{tabular}

bridge, England). $\mathrm{BF}_{3}$ in methanol was supplied by Sigma and sodium methoxide from Aldrich (Steinheim, Germany).

A stock solution of fatty acids was prepared by dissolving approximately $100 \mathrm{mg}$ of each fatty acid in $10 \mathrm{~mL}$ of methanol.

Samples. The samples were the monovarietal juices obtained from cider apples belonging to sweet and sharp varieties.

The apple varieties used in this study were grown in the experimental orchard of the S.E.R.I.D.A. (Villaviciosa, Asturias, northern Spain). Two different types of sweet cider apples, namely Coloradona and Verdialona, and four different types of sharp cider apples, Durona de Tresali, Xuanina, Raxao, and Solarina were chosen in this study as a result of their agronomical and technological suitability, since they present a reasonable resistance to fungi such as Venturia inaequaliz, Nectria galligena, Podosphaera leucotricha, and Monilia fructigena and ripen by the month of November, which is the usual period of cider-making in the north of Spain. Samples were taken in three consecutive harvests: twelve samples from the 1997 and 1998 harvests, respectively, and six samples from the 1999 harvest. Apples from each variety were milled with a hammer mill and pressed by means of a small hydraulic press; the apple musts obtained were then frozen at $-20{ }^{\circ} \mathrm{C}$ until analysis. Before analysis, the apple musts were defrosted overnight at $4{ }^{\circ} \mathrm{C}$. The samples were filtered and $50 \mu \mathrm{L}$ of a solution of internal standards was added.

Extraction of lipids from the processed juices was carried out using the Folch mixture (9). Apple juice $(50 \mathrm{~mL})$ was placed in a separator funnel and extracted with $60 \mathrm{~mL}$ of a mixture of chloroform and methanol $(2: 1, \mathrm{v}: \mathrm{v})$ by shaking for $5 \mathrm{~min}$. The organic and aqueous phases were separated by centrifugation. After filtration, the extracts were combined, dried on anhydrous sodium sulfate, and evaporated to dryness in a rotary evaporator at $30{ }^{\circ} \mathrm{C}$. Esterification of fatty acids was carried out on the extracts evaporated to dryness by adding $5 \mathrm{~mL}$ of $0.5 \mathrm{M} \mathrm{NaOH}$ and boiling for $30 \mathrm{~min}$. After addition of $\mathrm{BF}_{3}$ in methanol (5 $\mathrm{mL}$ ), the solution was boiled for $20 \mathrm{~min}$. After cooling, the fatty acid methyl esters were extracted with $3 \times 3 \mathrm{~mL}$ of hexane by shaking for $5 \mathrm{~min}$; the organic and aqueous phases being separated by centrifugation. The hexane extracts were combined, dried with anhydrous sodium sulfate, and transferred to a concentration tube; the extract being concentrated to $0.5 \mathrm{~mL}$ under a stream of nitrogen.

A Hewlett-Packard 5970 mass selective detector interfaced with a Hewlett-Packard 5790 gas chromatograph was used to identify the compounds by comparing retention times and mass spectra with those of standard methyl esters.

Quantification of fatty acids was performed by GC-FID (Unicam 610). The analytical conditions were as follows: column, acidified poly(ethylene glycol $)(30 \mathrm{~m} \times 0.25 \mathrm{~mm}, 0.25$ $\mu \mathrm{m}$ film thickness) MFE-1000; temperatures, initial isotherm of $80{ }^{\circ} \mathrm{C}$ for $1 \mathrm{~min}$, gradient, $5^{\circ} \mathrm{C} / \mathrm{min}$ from 80 to $230{ }^{\circ} \mathrm{C}$, final isotherm, $230{ }^{\circ} \mathrm{C}$ for $10 \mathrm{~min}$; carrier, nitrogen at a flow of 1.8 $\mathrm{mL} / \mathrm{min}$; injector temperature $290{ }^{\circ} \mathrm{C}$; detector temperature 300 ${ }^{\circ} \mathrm{C}$. Samples of $1 \mu \mathrm{L}$ were injected. Concentrations of fatty acid methyl esters were calculated by the internal standard method. An individual internal standard curve was generated for each fatty acid because of differences in response factors. Two internal standards were used: nonanoic (C9) and nonadecanoic acid (C19). Nonanoic acid was the most suitable one for the quantification of volatile and medium-chain fatty acids $(\mathrm{C} 8, \mathrm{C} 10$, $\mathrm{C} 12, \mathrm{C} 14)$ and nonadecanoic acid for the longer fatty acids (C15, $\left.\mathrm{C} 16, \mathrm{C} 16: 1^{\Delta 9}, \mathrm{C} 18, \mathrm{C} 18: 1^{\Delta 9}, \mathrm{C} 18: 2^{\Delta 9,12}\right)$. The concentration, expressed in $\mathrm{mg} / \mathrm{L}$, referred to juice.

Data Processing. The data were processed using the PARVUS statistical package (10). We used a data matrix with 30 observations (cider apple juices) and 10 chemical variables (fatty acids).

\section{RESULTS AND DISCUSSION}

Fatty Acids Analysis. Table 1 reports the mean values of fatty acids of ten sweet cider apple cultivars and twenty sharp cider apple cultivars. Ten fatty acids were quantified; two of these (palmitic and stearic acid) constituting the major fatty acids in apple juice. Saturated acids predominate over unsaturated acids. Among unsaturated acids, oleic predominates over linoleic and palmitoleic acid. Linolenic and arachidic acids are present in traces.

Statistical Analysis. Univariate Analysis. Before the chemometric techniques were used to typify cider apple cultivars according to their fatty acids content, a univariate analysis was carried out in order to know whether any of the fatty acids by themselves would allow us to differentiate the two categories established. The highest Fisher weight computed corresponded to linoleic acid (1.052). However, the use of the most discriminant variable did not allow us correctly to distinguish both technological categories, so multivariate treatment was needed.

Cluster Analysis. A selection of original variables was carried out in order to avoid collinearities between variables and to reduce noise in the models constructed. With this objective in mind, an exploratory cluster analysis of the variables was chosen. A Euclidean metric was used to determine similarities between variables and the Ward agglomerative method was employed to construct the dendrogram. We chose a similarity level of 0.7777 in order to decrease the heterogeneity of the variables. As can be seen in Figure 1, where the dendrogram of variables is displayed, a grouping between C14 and C12 (correlation coefficient significant at level 95\%, 0.8874) was detected at this similarity level; following a selection criterion at random, $\mathrm{C} 12$ was removed from the data matrix. Therefore, the new matrix was constructed with thirty observations and 


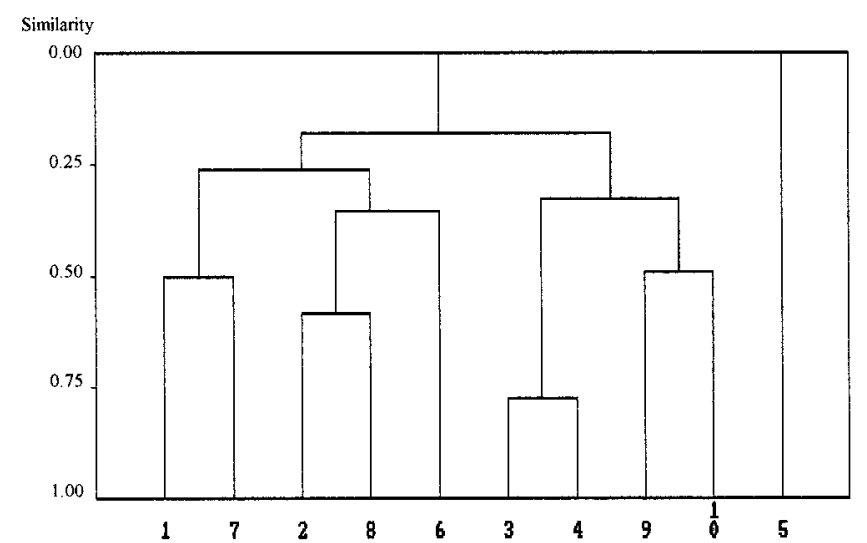

Figure 1. Dendrogram of variables: 1, caprylic; 2, capric; 3, lauric; 4, myristic; 5 , pentadecanoic; 6 , palmitic; 7 , palmitoleic; 8 , stearic; 9 , oleic; 10, linoleic acid.

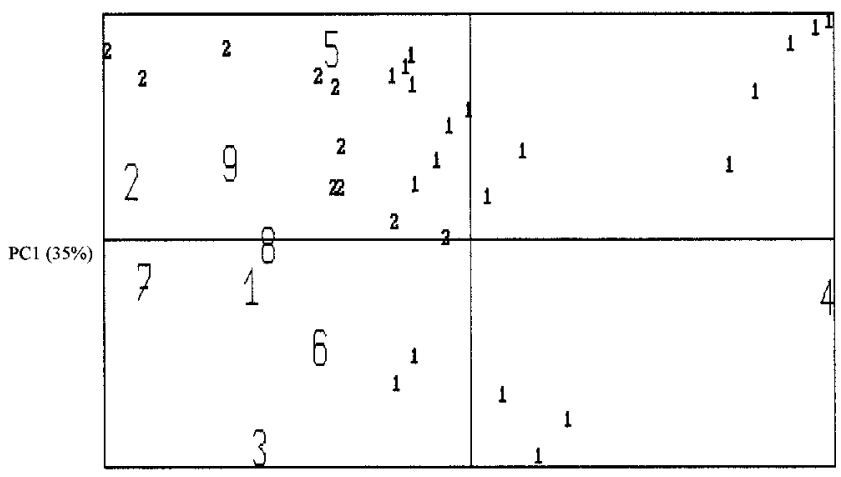

PC3 (17\%)

Figure 2. Projection of samples and variables on the factorial plane formed by the first and third components. (1) Sharp and (2) sweet juices; 1 , caprylic; 2, capric; 3, myristic; 4, pentadecanoic; 5, palmitic; 6 , palmitoleic; 7, stearic; 8, oleic; 9, linoleic acid.

nine variables (fatty acids: C8, C10, C14, C15, C16, C16:1 ${ }^{\Delta 9}$, $\mathrm{C} 18, \mathrm{C} 18: 1^{\Delta 9}$, and $\mathrm{C} 18: 2^{\Delta 9,12}$ ).

Principal Components Analysis. Once the selection of variables had been carried out, a principal components analysis (PCA) was carried out to detect the data structure and determine the relationships between samples and original variables. Five predictive components, which accounted for $92 \%$ of the variance, were computed from an internal procedure of validation, the full double-cross validation method, using five cancellation groups and $95 \%$ of the retained variance. The criterion followed for determining the number of predictive factors was the minimization of the prediction residual error square sum (PRESS).

Observations and variables were projected onto the factorial plane formed by the first and third components (retained variance 52\%) (Figure 2). As we can see in this figure, $90 \%$ of the samples belonging to the sweet category were placed in the upper left corner of the plane. At the same time, this figure shows that $\mathrm{C} 8, \mathrm{C} 10, \mathrm{C} 18, \mathrm{C} 18: 1^{\Delta 9}$, and $\mathrm{C} 18: 2^{\Delta 9,12}$ had negative scores with respect to the first component, and $\mathrm{C} 16$ had a positive score with respect to the third component. Thus, we can consider these fatty acids to be related to cider apple cultivars belonging to the sweet category; however, pentadecanoic acid, is related to the sharp category. As is well-known, unsaturated fatty acids containing one or more $(Z, Z)-1,4$ pentadienoic moieties, such as linoleic acid $\left(\mathrm{C} 18: 2^{\Delta 9,12}\right)$, can convert into "green note" flavor compounds such as C6-
Table 2. Classification Matrix by LDA Methoda

\begin{tabular}{cccc}
\hline & \multicolumn{2}{c}{ assigned category } & \\
\cline { 2 - 3 } true category & sharp & sweet & hits \\
\hline sharp & 19 & 1 & 95 \\
sweet & 1 & 9 & 90 \\
overall & & & 93
\end{tabular}

a Classification hits, $\%$.

Table 3. Prediction Matrix Using an Internal Validation Method with Five Cancellation Groups ${ }^{a}$

\begin{tabular}{cccc}
\hline & \multicolumn{2}{c}{ assigned category } & \\
\cline { 2 - 3 } true category & sharp & sweet & hits \\
\hline sharp & 17 & 3 & 85 \\
sweet & 2 & 8 & 80 \\
overall & & & 83
\end{tabular}

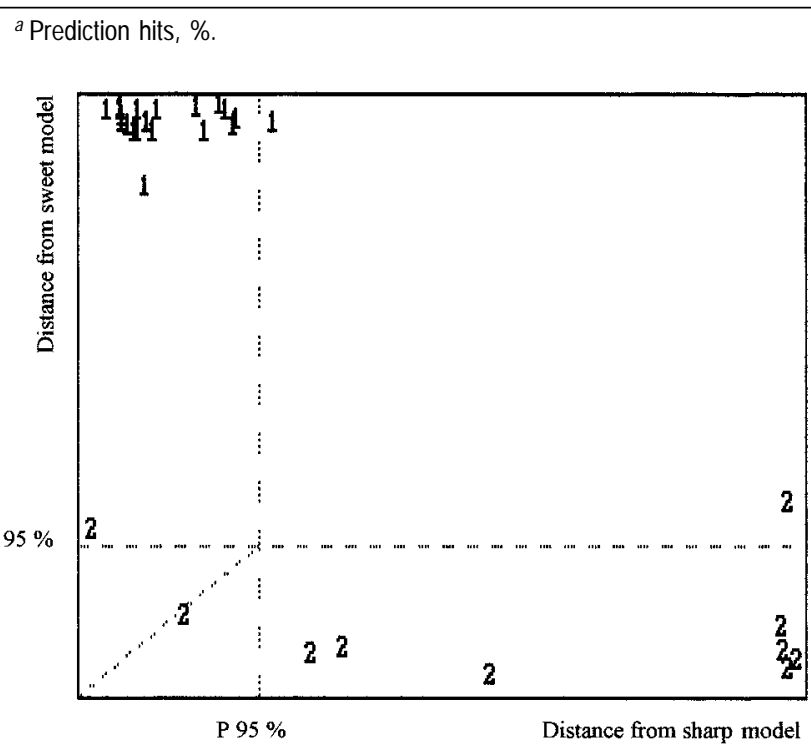

Figure 3. Coomans' diagram obtained from SIMCA analysis (1, sharp; 2, sweet category).

aldehydes and C6-alcohols by the action of lipoxygenase (LOX), oxygen, hydroperoxide lyase, and alcohol dehydrogenase. At the same time, the formation of methyl ketones from fatty acids such as $\mathrm{C} 8$ has been assumed; a $\beta$-oxidation mechanism being involved in this process. Therefore, it may be concluded that sweet cider apple varieties could make an important contribution to the final flavor of processed apple products as a result of the biochemical transformation of their lipid components. At the same time, the mixture of the cider apple varieties used in cider making might affect the fermentation of apple juice and the foam characteristics of the cider produced because of the initial level of fatty acids. Fatty acids such as caprylic (C8) and capric (C10) acid play a key role in causing arrested fermentations; and linoleic and oleic acid may inhibit foam formation at concentrations higher than $0.1 \mathrm{mg} / \mathrm{L}$. This is probably a consequence of a spreading effect that causes a local thinning of the film and its eventual breakage.

Linear Discriminant Analysis. From the data structure visualized on the factorial plane, we computed and validated a decision rule by means of a linear discriminant analysis (LDA) in order to classify cider apple varieties according to their fatty acids content. Validation was carried out from a cross validation method using five cancellation groups. Matrixes of classification and prediction are shown in Tables 2 and 3. As we can see, 
Table 4. Sensitivity (\%) and Specificity (\%) of Sharp and Sweet Classes According to the SIMCA Model

\begin{tabular}{lcr}
\hline & sensitivity & speci ficity \\
\hline class & & \\
sharp & 95 & 80 \\
sweet & 80 & 100 \\
\hline
\end{tabular}

hits were higher than $80 \%$ in all cases, so we may conclude that the mathematical rule constructed is sufficiently reliable and robust for classification purposes.

Soft Independent Modeling of Class Analogy (SIMCA). When the data matrix contains a reduced number of samples in relation to variables, a classification method such as SIMCA should be used. This chemometric technique shows interesting features, such as the principle of class modeling, computing a disjoint principal component model for each class. Four predictive components were computed for each class (sharp and sweet) using a SIMCA reduced model that accounted for $87 \%$ and $92 \%$ of the variance, respectively. Figure 3 shows a Coomans' diagram in which the two SIMCA boxes are displayed. Hits of classification were $93 \%$; $100 \%$ of the samples belonging to the sharp class and $80 \%$ of the samples belonging to the sweet category being correctly classified. Sensitivities and specificities of both models are shown in Table 4.

The SIMCA models computed were sufficiently sensitive and specific; the sharp model did not recognize $5 \%$ of its samples and accepted $20 \%$ of samples belonging to the sweet class, while the sweet class did not recognize $20 \%$ of its samples and did not accept any sample of the sharp category.

\section{CONCLUSIONS}

The use of multivariate techniques of data analysis, namely PCA, LDA, and SIMCA allowed us to ascertain the role played by some fatty acids in the discrimination between cider apple varieties belonging to sweet and sharp categories. The results obtained show that $\mathrm{C} 8, \mathrm{C} 10, \mathrm{C} 16, \mathrm{C} 18, \mathrm{C} 18: 1^{\Delta 9}$, and $\mathrm{C} 18: 2^{\Delta 9,12}$ are closely related to cider apple cultivars of the sweet category, while $\mathrm{C} 15$ is related to the sharp class.

\section{LITERATURE CITED}

(1) Ratledge, C.; Dickinson, F. M. Lipids and Fatty Acids as Potential Flavour Components using Microbial Systems. Bioflavour95 1995, 153-166.

(2) Geneix, C.; Lafon-Lafourcade, S.; Ribéreau-Gayon, P. Effect des Acides Gras sur la Viabilité des Populations de Saccharomyces cerevisiae. C. R. Acad. Sci. 1983, 296, 943-947.

(3) Lafon-Lafourcade, S.; Geneix, C.; Ribéreau-Gayon, P. Inhibition of Alcoholic Fermentation of Grape Must by Fatty Acids Produced by Yeast and their Elimination by Yeast Ghosts. Appl. Environ. Microbiol. 1984, 47, 1246-1249.

(4) MacLeod, A. M. Beer. In Alcoholic Beverages; A. H. Rose, Ed.; Academic Press: London, 1977.

(5) García-López, C.; Grané-Teruel, N.; Berenguer-Navarro, V.; García-García, J.; Martín-Carratalá, M. L.. Major Fatty Acid Composition of 19 Almond Cultivars of Different Origins. A Chemometric Approach. J. Agric. Food Chem. 1996, 44, 17511755.

(6) Martín-Carratalá, M. L.; García-López, C.; Berenguer-Navarro, V.; Grané-Teruel, N. New Contribution to the Chemometric Characterization of Almond Cultivars on the Basis of Their Fatty Acid Profiles. J. Agric. Food Chem. 1998, 46, 963-967.

(7) Arena, E.; Campisi, S.; Fallico, B.; Maccarone, E. Fatty Acids of Italian Blood Orange Juices. J. Agric. Food Chem. 1998, 46, 4138-4143.

(8) Blanco-Gomis, D.; Mangas Alonso, J. J.; Margolles Cabrales, I.; Arias Abrodo, P. Gas Chromatographic Analysis of Total Fatty Acids in Cider. J. Agric. Food Chem. 2001, 49, 12601263.

(9) Ferrari, G.; Meunier, J. M.; Feuillat, M. Determination of Wine and Yeast Fatty Acids. Sci. Aliments 1987, 7, 61-76.

(10) Forina, M.; Leardi, R.; Lanteri, S.; Armanino, C. In PARVUS. An Extendable Package of Programs for Data Exploration, Classification and Correlation; Elsevier: Amsterdam, 1998.

Received for review July 30, 2001. Revised manuscript received November 20, 2001. Accepted November 25, 2001. This work was made possible by financial support from the CICYT (ALI 96-1219-C02-02). We thank Dr. Enrique Dapena (S.E.R.I.D.A., Villaviciosa, Spain) for supplying cider apple juices.

JF010998X 